\title{
Metalation of Porphyrins by Lanthanide Atoms at Interfaces: Direct Observation and Stimulation of Cerium Coordination to 2H-TPP/ ${ }_{3} \mathrm{Ag}(111)$
}

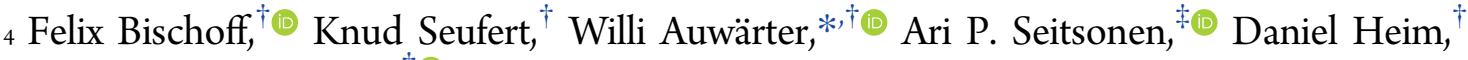 \\ $s$ and Johannes V. Barth ${ }^{\dagger}$ \\ $6{ }^{\dagger}$ Physik-Department E20, Technische Universität München, James Franck Straße 1, D-85748 Garching, Germany \\ 7 Département de Chimie, Ecole Normale Supérieure, Sorbonne Université, Centre National de la Recherche Scientifique, 24 rue \\ 8 Lhomond, F-75005 Paris, France
}

ABSTRACT: Although on-surface metalation protocols of tetrapyrroles with $3 d$ metals are well established, reports on the formation of lanthanide tetrapyrrole complexes are scarce. Here, we address the synthesis of lanthanide-tetrapyrrole units in detail, refining earlier findings. Specifically, the formation of cerium tetraphenylporphyrin (Ce-TPP) was induced on $\mathrm{Ag}(111)$ either by thermal annealing or by a manipulation procedure using a scanning tunneling microscope (STM) tip. While the self-assembled TPP arrays are not altered upon Ce metalation, our STM observations show distinct modifications of submolecular features reflecting a multistep reaction pathway. The metalation proceeds from an initial configuration with a $2 \mathrm{H}-$ TPP molecule sitting atop a Ce atom via an intermediate state, where the macrocycle is partially deprotonated, to metalated Ce-TPP. The hitherto
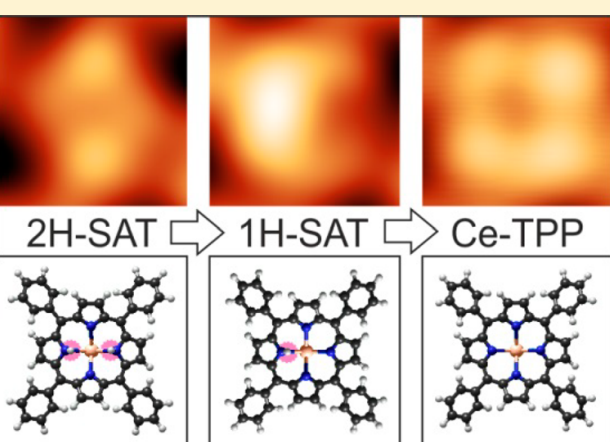
elusive $1 \mathrm{H}$ species state-hypothesized in several $3 \mathrm{~d}$ metalation studies-is visualized directly. Our study provides novel insights into the on-surface synthesis of tetrapyrroles and lanthanide-based nanosystems.

\section{INTRODUCTION}

26 On-surface tetrapyrrole metalation protocols under ultrahigh 27 vacuum (UHV) have been established as versatile approaches 28 for the engineering of metal-organic complexes and nanostruc29 tures that cannot be easily achieved on surfaces by standard 30 preparation techniques. ${ }^{1-4}$ The considerable research efforts 31 are motivated by the potential for applications of metallo32 tetrapyrroles in photovoltaics, ${ }^{5,6}$ catalysis, ${ }^{7}$ sensing ${ }^{8}$ and 33 quantum technology, among many others. ${ }^{9}$ Since the metal 34 center in the macrocycle has great influence on molecular 35 functionalities, the selection and control over this central ion is 36 essential. While the formation of $3 d$ metal tetrapyrrole 37 complexes was intensely studied, ${ }^{1,2,10,11}$ reports on the in situ 38 incorporation of lanthanide metals are scarce and emphasize 39 sandwich structures where the lanthanide is bound not to one 40 but to two tetrapyrroles ${ }^{12}$ and thin films thereof. ${ }^{13}$ Double41 deckers or multideckers where 8 -fold coordination of 42 lanthanide centers couples two tetrapyrrole macrocycles are 43 of great interest and have been explored for many years. ${ }^{14,15}$ 44 They can notably act as field-effect transistors and single45 molecule magnets, ${ }^{16-21}$ in favorable situations preserving their 46 properties even on metallic supports. ${ }^{22,23}$ However, to gain a 47 fundamental understanding of the relevant chemical processes 48 during on-surface metalation and to tackle surface-anchored 49 coordinatively unsaturated lanthanide tetrapyrrole complexes, the investigation of species comprising only one molecular 50 ligand is desirable. Solution-based approaches and theoretical 51 studies describe the redox reaction from free-base to metalated 52 molecule as a multistep sequence for transition metals. ${ }^{24-26} 53$ First, the intact macrocycle binds to a metal ion. This precursor 54 state is often referred to as sitting atop (SAT) complex. 55 Subsequently, one hydrogen is removed from the macrocycle to 56 form an intermediate SAT and finally, the metallotetrapyrrole 57 evolves under release of $\mathrm{H}_{2}$. This theoretically suggested 58 reaction pathway has been experimentally corroborated by 59 observing the precursor in solution ${ }^{27}$ as well as at the solid- 60 vacuum interface. ${ }^{28-31}$ To date a clear experimental proof is 61 still lacking for the intermediate SAT.

Herein, we report a low-temperature (LT) STM and 63 scanning tunneling spectroscopy (STS) study on intermediate 64 complexes formed during the metalation of tetrapyrroles with 65 lanthanide atoms, complemented by extensive density func- 66 tional theory (DFT) calculations. Specifically, we use free-base 67 5,10,15,20-tetraphenylporphyrin (2H-TPP) molecules and Ce 68 deposited sequentially on $\mathrm{Ag}(111)$. Both annealing and STM 69 tip manipulations trigger the stepwise metalation reaction from 70

Received: October 19, 2017

Revised: January 28, 2018 

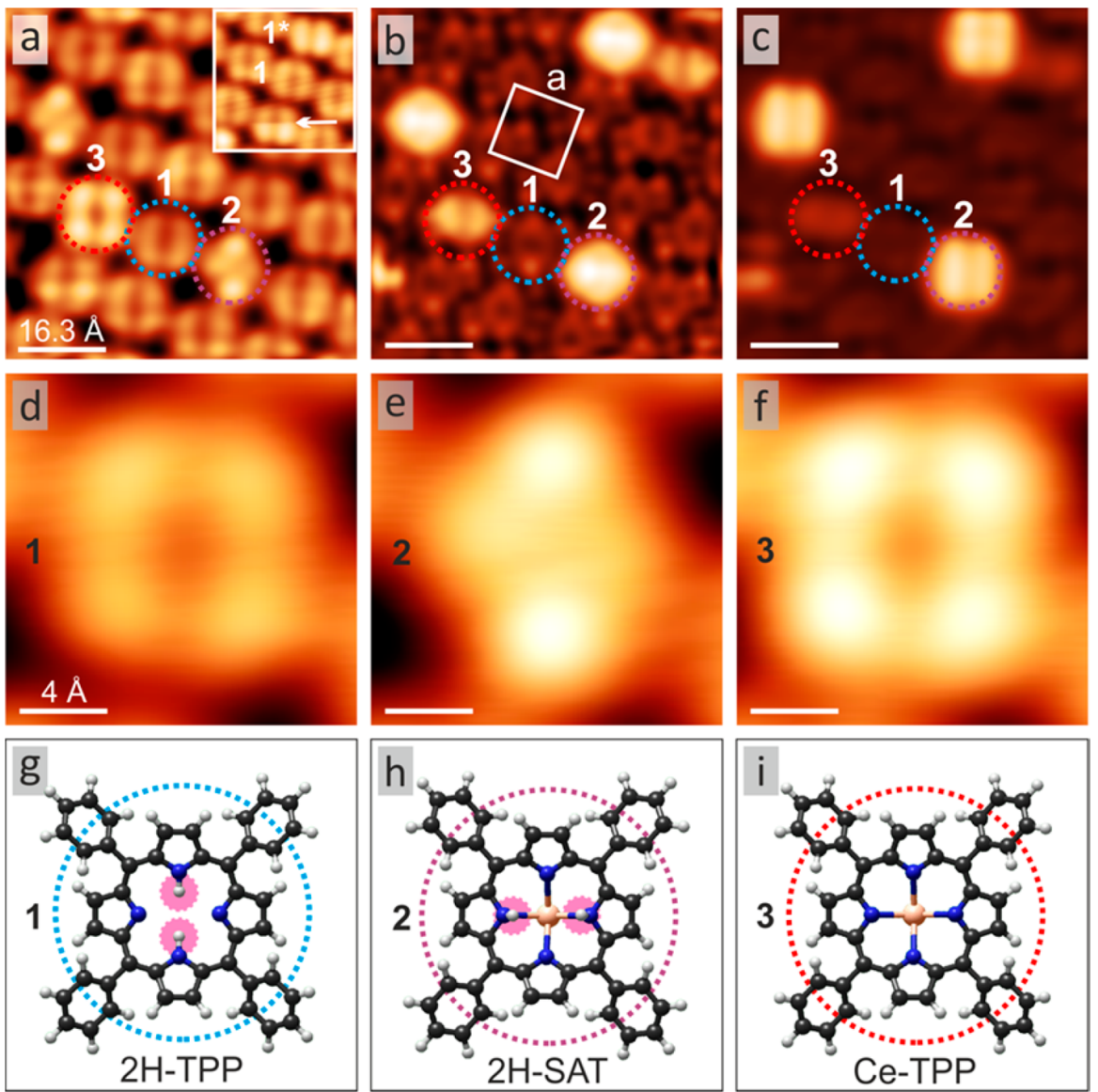

Figure 1. STM characterization of Ce-induced conversions within TPP arrays (measured with (a) $U=1.25 \mathrm{~V}$, (b) $U=-0.05 \mathrm{~V}$, and (c) $U=-1.25$ $\mathrm{V}$; all images of the same area, the white square in part b) highlights the TPP $/ \operatorname{Ag}(111)$ unit cell with side length $a=1.4 \mathrm{~nm})$. At $1.25 \mathrm{~V}$ the three prevalent species 1-3 exhibit a similar apparent height and are easy to distinguish. $\mathbf{1}$ is 2 H-TPP (blue), 2 a precursor sitting atop complex with a cerium atom underneath the tetrapyrrole macrocycle (purple) and 3 the metalated Ce-TPP following hydrogen abstraction (red). The inset in part a depicts the two tautomeric forms $\mathbf{1}$ and $\mathbf{1}^{*}$ of $2 \mathrm{H}$-TPP. The white arrow serves as guide to the eye for a scanning induced tautomerization reaction. $(\mathrm{d}-\mathrm{f})$ Detailed images of each species at $1.25 \mathrm{~V} .(\mathrm{g}-\mathrm{i})$ Corresponding illustrative models. In the models, the central hydrogens are highlighted for clarity. The orientation of the models corresponds directly to the STM data shown above and was determined by simulated STM images based on DFT modeling (cf. Figure 5). Scan parameters: $(\mathrm{a}, \mathrm{d}-\mathrm{f}) I=0.20 \mathrm{nA}, U=1.25 \mathrm{~V} ;(\mathrm{b}) I=0.20 \mathrm{nA}, U=-0.05 \mathrm{~V} ;(\mathrm{c}) I=0.20 \mathrm{nA}, U=-1.25 \mathrm{~V}$.

71 the precursor $2 \mathrm{H}$-SAT, via the reaction intermediate $1 \mathrm{H}-\mathrm{SAT}$

72 to the metalloporphyrin unit. This study covers the complete

73 on-surface synthesis scheme toward formation of Ce-TPP. A

74 theoretical examination of the energetics of the observed

75 species based on DFT modeling including the surface

76 rationalizes the experimental findings and a comparison of

77 simulated STM images helps to identify all involved species.

78 DFT also allows to follow subtle geometric adaptations during

79 the metalation process. Furthermore, we reassess previously

80 published data on the interaction of Ce with porphyrin arrays. ${ }^{32}$

81 Whereas these earlier findings suggested Ce-TPP formation

82 within mixed cobalt (II) tetraphenylporphyrin (Co-TPP) and

83 2H-TPP precursor layers, they are now associated with a more

84 complex $\mathrm{Ce}-\mathrm{Co}-\mathrm{TPP}$ structure involving $3 \mathrm{~d}-4 \mathrm{f}$ metal

85 interactions (see also the related finding of site-specific $\mathrm{Fe}$

86 atoms bonding at adsorbed Co-TPP layers ${ }^{33}$ ).

\section{METHODS}

Scanning Tunneling Microscopy. All STM experiments 89 were performed in a custom-designed UHV system providing a 90 base pressure below $5 \times 10^{-10} \mathrm{mbar}^{34}$ All data were acquired 91 employing a low-temperature CreaTec-STM ${ }^{35}$ with the sample 92 held at $6 \mathrm{~K}$ using electrochemically etched W tips. For imaging the constant-current mode was employed. In the figure 93 captions, $U$ refers to the bias voltage applied to the sample. 94 Models were generated in the framework of the HyperChem 95 7.5 Software Package. The Gwyddion software was used for the 96 analysis of STM images. ${ }^{36}$

Sample Preparation. The monocrystalline $\operatorname{Ag}(111) 98$ substrate was cleaned by repeated $\mathrm{Ar}^{+}$sputtering cycles at an 99 energy of $800 \mathrm{eV}$, followed by annealing at $730 \mathrm{~K}$ for $10 \mathrm{~min} .100$ The synthesis of mixed 2H-TPP/2H-SAT/Ce-TPP arrays was 101 realized by depositing 2H-TPP (Sigma-Aldrich, purity $\geq 99 \%$ ) 102 by organic molecular beam epitaxy from a thoroughly degassed 103 quartz crucible held at $600 \mathrm{~K}$ and evaporation of Ce atoms from 104 a homemade water-cooled cell by resistively heating a W 105 filament enclosing a Ce ball of high purity (99.9999\%, MaTecK 106 GmbH, D-52428 Jülich, Germany). During deposition, the 107 $\mathrm{Ag}(111)$ surface was kept at $373 \mathrm{~K}$. For the control experiment 108 with Co-TPP (Sigma-Aldrich, purity 95\%), Co-TPP was 109 deposited from a thoroughly degassed quartz crucible held at 110 $630 \mathrm{~K}$. During the growth experiments the pressure remained 111 $<5 \times 10^{-10}$ mbar. Regarding coverage, we define one 112 monolayer as a surface fully covered by $2 \mathrm{H}$-TPP species. 113

Density Functional Theory. We performed total energy 114 calculations using DFT $^{37}$ within the Kohn-Sham formalism ${ }^{38} 115$ 
116 using the Quantum ESPRESSO code (http://www.Quantum117 ESPRESSO.org/). ${ }^{39}$ The rB86-vdW-DF2 approximation ${ }^{40}$ to 118 the exchange correlation functional was applied. We used the 119 cutoff energy of 60 and 350 Ry to expand the Kohn-Sham 120 orbitals and the augmented electron density, respectively. The 121 projector-augmented wave data sets were obtained from 122 pslibrary. ${ }^{41} \Gamma$ point was used in the calculations due to the 123 relatively large dimensions, together with the Fermi-Dirac 124 broadening of the occupation numbers with a width of $50 \mathrm{meV}$. 125 Two molecules were inserted in $\mathrm{a}(7 \times 4 \sqrt{3})$ unit cell that is 126 close to the experimentally derived periodicity. ${ }^{42}$ Five layers of 127 substrate were employed. The computed lattice constant of $1284.1075 \AA$ was used in the calculations. The initial structure was 129 constructed following the literature, ${ }^{42}$ with the centers of 130 molecules at two hollow sites (one hcp, one fcc site), and 131 subsequently relaxing the geometry. The centers moved upon 132 the relaxation somewhat, but remained at about the same 133 distance from their corresponding 3-fold site. The adsorption 134 energy was defined as the difference in the total energies of the 135 adsorbed complex minus the relaxed clean surface and two 136 times an individual molecule in the gas phase, with the 137 convention of positive adsorption energy indicating attraction. 138 The simulated STM images were obtained using the Tersoff139 Hamann method $^{43}$ by including the orbital densities of the 140 orbitals from the Fermi energy to the given bias voltage, and 141 using an iso-value $\left(10^{-7} \mathrm{e} / \mathrm{a}_{0}^{3}\right)$ of the partial electron density at 142 each lateral point on the density mesh to construct the two143 dimensional maps.

\section{EXPERIMENTAL AND THEORETICAL RESULTS}

145 STM and STS Observations. meso-Substituted tetraphe146 nylporphyrins (TPP) are known to assemble into extended, 147 well ordered islands on the (111) surface of a silver single 148 crystal. $^{42,44}$ Irrespective of the exposure to small amounts of Ce 149 atoms, the molecules retain the pertaining noncommensurate 150 lattice with a square unit cell $(1.4 \mathrm{~nm} \times 1.4 \mathrm{~nm})$, marked in 151 white in Figure $1 \mathrm{~b}$ and Figure S1c. Each molecule is oriented 152 with its main axis along a $\langle 11 \overline{2}\rangle$ high symmetry direction of the $153 \mathrm{Ag}(111)$ surface (for details see Supporting Information). At 154 submonolayer coverages, the island formation is mainly driven 155 by attractive T-type interactions between the phenyl meso156 substituents. ${ }^{45}$ 2H-TPPs appear as described in previous STM 157 studies: ${ }^{46-48}$ a donut-shaped macrocycle appears at negative 158 sample bias and at small bias voltages around the Fermi level, 159 while a window-like 4-fold symmetric contrast dominates when 160 probing the unoccupied states (Figure 1 and Figure S1). A 2H161 TPP unit is marked by blue dashed circles and is referred to as 162 species 1 in Figure 1. Scanning at elevated bias voltages (IUI > $1630.8 \mathrm{~V}$ ) induces reversible switching of contrast as indicated by 164 the white arrow in the inset in Figure 1a. This reversible 165 transition is assigned to a tautomerization reaction, i.e., the 166 correlated proton transfer inside the macrocycle between the 167 nitrogen atoms. ${ }^{48-51}$ Consequently, 2H-TPP can appear as two 168 tautomers 1 and $\mathbf{1}^{*}$

169 After exposing submonolayer $2 \mathrm{H}$-TPP arrays on $\mathrm{Ag}(111)$ to 170 an atomic beam of $\mathrm{Ce}$ at $330 \mathrm{~K}$, intramolecular changes are 171 discernible in the STM images (Figure 1), whereby the order in 172 the molecular layer is unperturbed. Three different species can 173 be identified: free-base TPP (1: 2H-TPP, blue), intact 2H-TPP 174 sitting atop of a single cerium atom (2: $2 \mathrm{H}-\mathrm{SAT}$, purple) and 175 the metalated Ce-TPP (3, red). The detailed reasoning of these 176 assignments is elaborated below, notably in the computational 177 modeling (link to section computational modeling) and discussion sections (vide infra). Structure models of the species 178 are depicted in Figure $1 \mathrm{~g}-\mathrm{i}$. The initial SAT complex can be 179 discriminated by two prominent protrusions along a central axis 180 of the molecule for $U>1 \mathrm{~V}$ (see Figure 1a,e) and by its 181 increased apparent height for $U<-0.65 \mathrm{~V}$ featuring two 182 elongated lobes (purple circle in Figure 1c). Around the Fermi 183 level, 2H-SAT exhibits the largest apparent height and 184 dominates the image contrast (Figure 1b). Ce-TPP strongly 185 resembles $2 \mathrm{H}$-TPP but shows a larger apparent height $(\Delta z \approx 186$ $0.3 \AA$ A) for all voltages (cf. Figure 1d-f). An increased 187 adsorption height is confirmed by DFT simulations for all 188 cerium induced species giving values of $0.28 \AA$ (Ce-TPP), 0.42189 $\AA$ (2H-SAT) and $0.36 \AA$ for the 1 H-SAT identified and 190 discussed below (see also Table 3 ).

191

To further characterize the species and elucidate the origin of 192 the different submolecular contrast, STS and $\mathrm{d} I / \mathrm{d} V$ mapping of 193 prominent electronic contributions were carried out. The 194 results are depicted in Figure 2. A common feature in all spectra $195 \mathrm{f} 2$ is an increased density of states at $\approx 110 \mathrm{mV}$ (dashed line in 196 Figure 2a) that was observed consistently with different tips 197 and in different preparations (see also Figure S4a). Since it 198 appears at a comparable energy for all species within the same 199 array, it presumably does not reflect a molecular contribution, 200 but rather stems from an up-shifted surface state known for 201
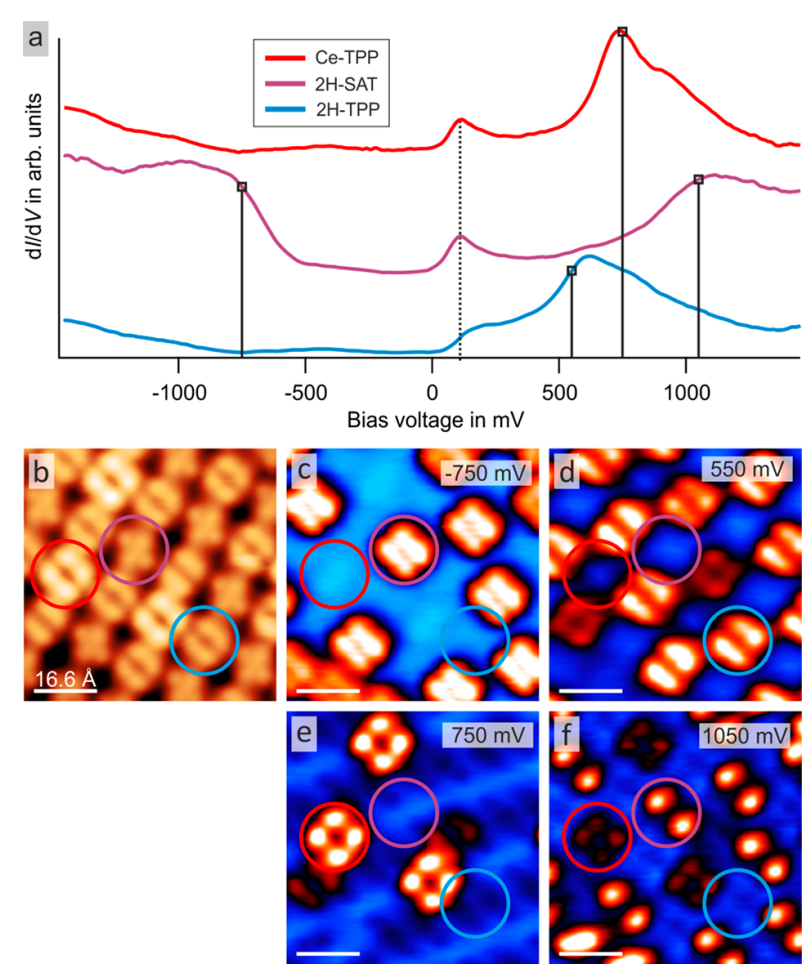

Figure 2. Electronic structure of $2 \mathrm{H}$-TPP and Ce-modified TPP. (a) STS of 2H-TPP, $2 \mathrm{H}$-SAT and Ce-TPP (set point: $I=0.20 \mathrm{nA}, U=$ $1.20 \mathrm{~V}$; color coding of marked species as in Figure 1). The spectra are offset for better comparison and represent averages of multiple measurements acquired above different molecules and positions within an array. Vertical lines mark biases selected for $\mathrm{d} I / \mathrm{d} V$ mapping in the array whose STM topography is depicted in part b $(I=0.20 \mathrm{nA}, U=$ $1.05 \mathrm{~V}) .(\mathrm{c}-\mathrm{f}) \mathrm{d} I / \mathrm{d} V$ maps of the spatial electron density distribution associated with molecular orbitals of different species: (c) 2H-SAT HOMO, (d) 2H-TPP LUMO, (e) Ce-TPP LUMO, and (f) $2 \mathrm{H}-\mathrm{SAT}$ LUMO. The bias is indicated in the top right of each map; current set point: $I=0.20 \mathrm{nA}$. 
202 weakly adsorbed overlayers on transition metal surfaces. ${ }^{52}$ The 203 unoccupied region $(U>0 \mathrm{~V})$, however, clearly shows distinct 204 molecular features. As expected for $2 \mathrm{H}-\mathrm{TPP}^{48,53}$ (blue curve in 205 Figure 2a), a broad peak from the lowest unoccupied molecular 206 orbital (LUMO) is located slightly above $600 \mathrm{mV}$.

207 For the Ce-modified species, the LUMO shifts to higher bias 208 voltages, specifically to $\approx 1050 \mathrm{mV}$ for $2 \mathrm{H}$-SAT (purple) and $209 \approx 750 \mathrm{mV}$ for Ce-TPP (red). In the occupied region $(U<0 \mathrm{~V})$, $2102 \mathrm{H}-\mathrm{TPP}$ and Ce-TPP do not exhibit molecular resonances for 211 the probed voltages down to $U=-2000 \mathrm{mV}$. By contrast, $2 \mathrm{H}-$ 212 SAT features a distinct resonance from the highest occupied 213 molecular orbital (HOMO) at $\approx-750 \mathrm{mV}$. The $\mathrm{d} I / \mathrm{d} V$ maps in 214 Figure $2 \mathrm{c}-\mathrm{f}$ furthermore present the spatial extent of the 215 observed molecular resonances. For comparison, Figure $2 b$ 216 shows an STM image of the same region with highlighted 217 molecules (blue, 2H-TPP; purple, 2H-SAT; red, Ce-TPP). The 218 2H-TPP LUMO extends as two elongated protrusions, 2 -fold 219 symmetrically across the iminic pyrroles, i.e. parallel to the main 220 molecular axis (Figure 2d). In addition, 2H-SAT and Ce-TPP 221 LUMO appear fundamentally different: 2H-SAT shows two 222 prominent protrusions (Figure $2 \mathrm{f}$ ) located on two opposite 223 macrocyclic pyrroles, while the Ce-TPP appears 4-fold 224 symmetric (Figure 2e). Moreover, it is interesting to note 225 that the 2H-SAT HOMO (Figure 2c) resembles the 2H-TPP 226 LUMO in shape and spatial extent.

227 In the data set, $2 \mathrm{H}$-SAT and Ce-TPP coexist, which indicates 228 that the employed preparation conditions did either not afford 229 complete conversion before cooling the sample or that $2 \mathrm{H}$-SAT 230 and Ce-TPP are equally favorable in the reaction pathway. The 231 latter option can be disregarded as the yield of fully metalated 232 porphyrins increases substantially following further sample 233 anneal to $390 \mathrm{~K}$ for $5 \mathrm{~min}$ with a conversion of approximately 234 90\% 2H-SAT into Ce-TPP.

235 The metalation reaction can also be triggered by scanning at 236 elevated bias and through voltage pulses. Interestingly, such 237 experiments give rise to a new species with only one bright lobe 238 (Figure 3). This species is tentatively assigned to an 239 intermediate SAT complex with a partially deprotonated 240 macrocycle. Accordingly, it is referred to as $1 \mathrm{H}-\mathrm{SAT}$, and can 241 be seen as intermediate species between the initial 2H-SAT and 242 the final Ce-TPP product. Indeed, it can finally be transformed 243 to Ce-TPP (cf. Figure 4). Figure 3 depicts the 1H-SAT STM 244 appearance (green circle). It can clearly be discriminated from 245 the other species by scanning at positive and negative sample 246 bias voltages as depicted in Figure 3a,b. For both voltage 247 regimes, 1H-SAT features the distinct off-centered lobe with 248 similar shape. In contrast, the other species change 249 fundamentally when reversing the bias polarity (cf. Figure 1).

250 A conversion of $2 \mathrm{H}$-SAT via $1 \mathrm{H}$-SAT to Ce-TPP can be 251 induced by applying bias voltages exceeding a threshold of $U \geq$ $2521.4 \mathrm{~V}$, or $U \leq-1.9 \mathrm{~V}$. Parts a-c of Figure 4 demonstrate the 253 stepwise, tip-induced metalation by applying a bias of $1.5 \mathrm{~V}$. 254 Below the given voltage the reaction intermediate is stable and 255 can be characterized by STM. Furthermore, the reaction can be 256 directly monitored in the current vs. time trace $I(t)$ of a voltage 257 pulse (Figure 4d). Along the lower trace (green) two steps in 258 the current signal are discernible, while the upper curve (red) 259 only shows one. A comparison of the STM images before and 260 after the pulses thus clearly testifies the formation of Ce-TPP. 261 Therefore, the single step does not correspond to a single 262 deprotonation, but to the full metalation reaction. Note that the 263 deprotonation does not necessarily correspond to a current 264 drop, but can likewise manifest as increase in the magnitude of
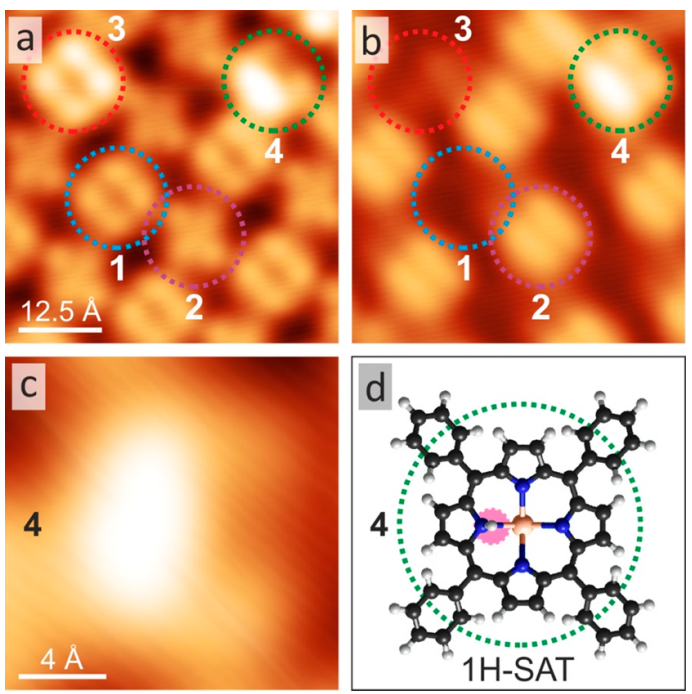

Figure 3. Intermediate Ce-tetrapyrrole complex 1H-SAT. 1H-SAT (4, green) appearing markedly different from species $1-3$, both at positive $(\mathrm{a} ; U=1.00 \mathrm{~V})$ and negative bias $(\mathrm{b} ; U=-0.65 \mathrm{~V})$. It exhibits one bright, off-centered lobe. Upon polarity change, 1-3 differ completely in STM data; 4 however remains alike in both voltage regimes. (c) Zoom onto $1 \mathrm{H}-\mathrm{SAT}$ and (d) corresponding structure model. The orientation of the model corresponds directly to the STM data next to it and was determined by simulated STM images based on DFT modeling (cf. following section). Scan parameters: (a, c) $I=0.20$ $\mathrm{nA}, U=1.00 \mathrm{~V}$; (b) $I=0.20 \mathrm{nA}, U=-0.65 \mathrm{~V}$.
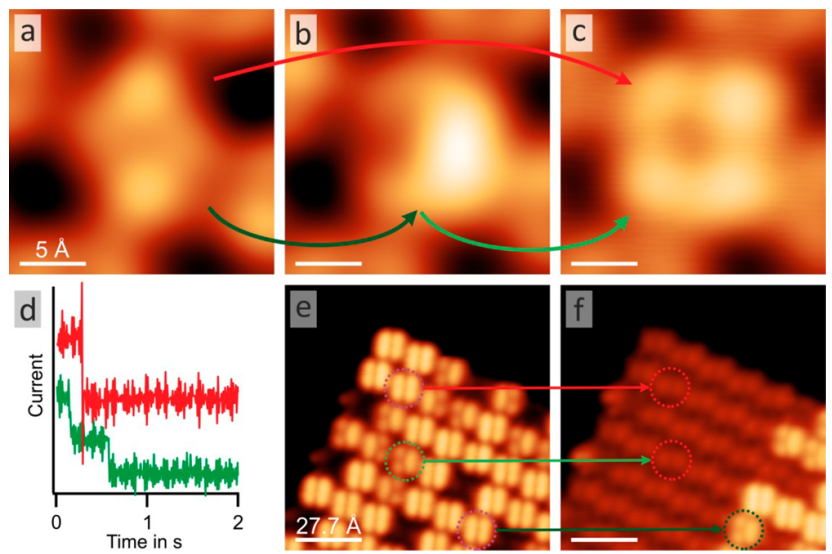

Figure 4. Tip-induced metalation reaction of $2 \mathrm{H}$-TPP with a single $\mathrm{Ce}$ atom. $(\mathrm{a}-\mathrm{c})$ The precursor complex $2 \mathrm{H}-\mathrm{SAT}(\mathrm{a})$ is singly deprotonated to form the reaction intermediate $1 \mathrm{H}-\mathrm{SAT}(\mathrm{b})$ and finally Ce-TPP (c) by applying a voltage of $1.5 \mathrm{~V}$. The red and green arrows depict the reaction pathways observable after tip-interactions. (d) The deprotonation/metalation can be monitored in the current vs time traces of voltage pulses as single (red) and double steps (green, traces vertically offset for clarity). (e, f) Applying a voltage of $2 \mathrm{~V}$ at the upper left corner of the array induces transformations of Ce-derived species proceeding simultaneously within the probed area. All possible reaction steps are observable: $2 \mathrm{H}$-SAT to Ce-TPP (top, red), $1 \mathrm{H}-\mathrm{SAT}$ to Ce-TPP (middle, light green), and 2H-SAT to 1H-SAT (bottom, dark green). Scan parameters: $(\mathrm{a}-\mathrm{c}) I=0.20 \mathrm{nA}, U=1.20 \mathrm{~V}$; $(\mathrm{e}, \mathrm{f}) I=$ $0.21 \mathrm{nA}, U=-1.02 \mathrm{~V}$.

the tunneling current, depending on the position of the tip 265 above the molecule.

The metalation reaction can be triggered locally with 267 molecular precision when applying pulses close to the threshold 268 


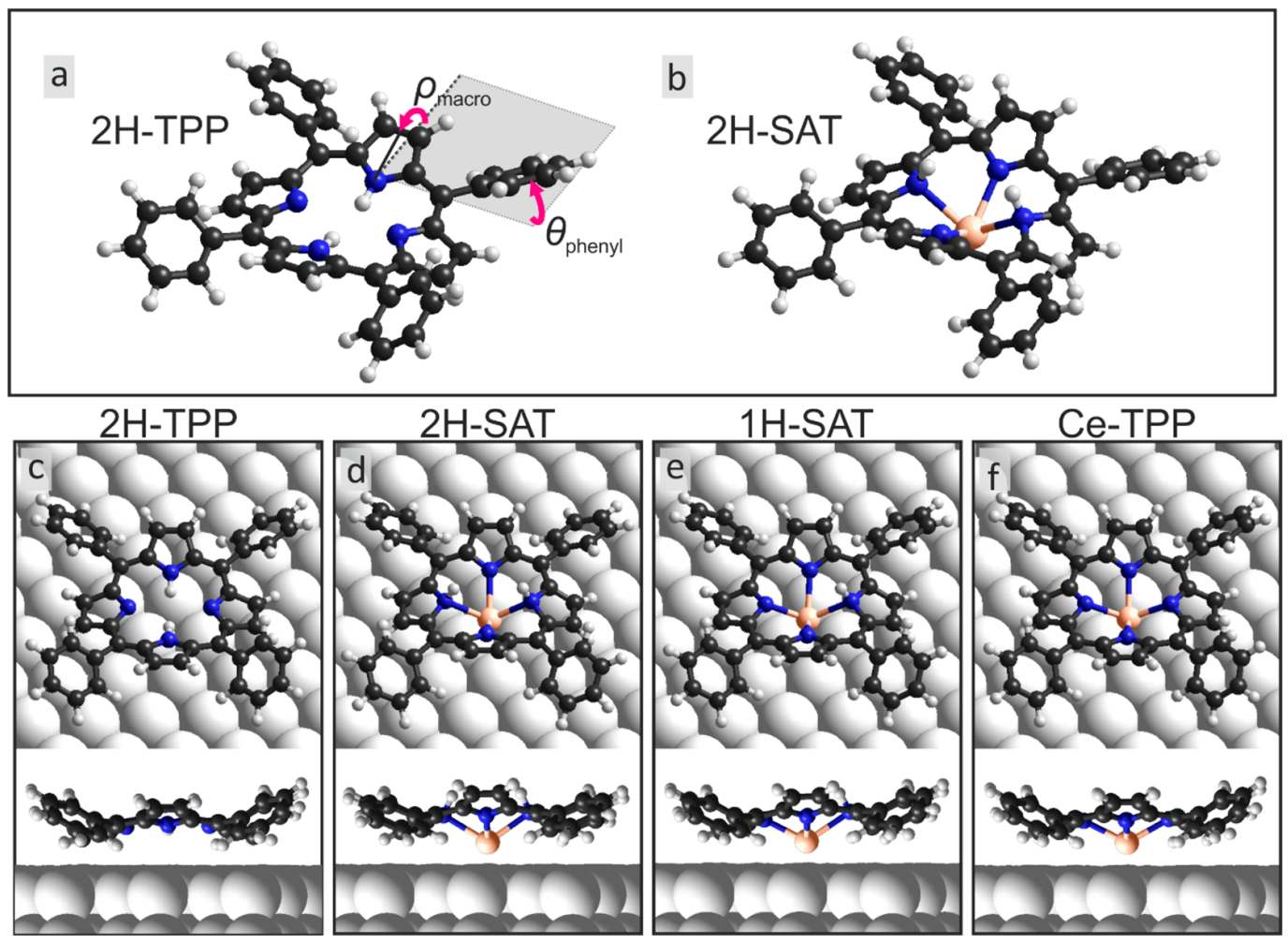

Figure 5. DFT-optimized structure and bonding of free-base, SAT and metalated TPP species on $\mathrm{Ag}(111)$. In part a, the macrocycle distortions and phenyl substituent rotational degree of freedom are indicated. The perspective view of the 2 H-SAT complex in part b visualizes intramolecular distortions and placement of protons. Parts $\mathrm{c}-\mathrm{f}$ display top and side views of educt, product, and SAT intermediate species.

269 value. Moreover, it can be induced over a large area with 270 increased bias voltage. The specific reaction trigger however 271 remains elusive and was not investigated systematically. Figure $2724 \mathrm{e}, \mathrm{f}$ demonstrate the simultaneous metalation of more than 25 273 molecules within a molecular SAT array by applying $2 \mathrm{~V}$ at the 274 top left corner of the island. The observed reactions include all 275 possible reaction steps indicated by arrows in Figure 3e,f: $2 \mathrm{H}-$ 276 SAT to Ce-TPP (red, top), 1H-SAT to Ce-TPP (bright green, 277 middle), and 2H-SAT to 1 H-SAT (dark green, bottom).

278 Computational Modeling. To rationalize the experimen279 tal findings, we performed total energy calculations using DFT 280 within the Kohn-Sham formalism. The calculations were 281 carried out on a $(7 \times 4 \sqrt{ } 3)$ unit cell with two molecules per 282 unit cell (cf. Figure S2). A visual overview of the results and 283 terminology is provided by the models depicted in Figure 5. 284 Tables 1 and 2 summarize the relevant energetics. Table 1 285 gives the adsorption energies of the two $2 \mathrm{H}$-TPP tautomers as

Table 1. Adsorption Energies of 2H-TPP and Ce-TPP Species from DFT Calculations ${ }^{a}$

$\begin{array}{lc}\quad \text { species } & \begin{array}{c}\text { adsorption energy } E_{\text {ads }} \\ (\mathrm{eV} / \text { molecule })\end{array} \\ \text { 2H-TPP, tautomer } \mathbf{1} & 3.22 \\ \text { 2H-TPP, tautomer } \mathbf{1}^{*} & 3.14 \\ \quad\left(\text { hydrogens rotated } 90^{\circ}\right) & 5.09 \\ \text { Ce-TPP, Ce down } & 3.46 \\ \text { Ce-TPP, Ce up } & \end{array}$

${ }^{a}$ For $2 \mathrm{H}$-TPP both tautomers were calculated, for Ce-TPP the two possible configurations for the cerium position above (Ce up) and below (Ce down) the macrocycle plane. Bold values are energetically favored.
Table 2. Binding Energy of Hydrogen Atoms in 2H-SAT and 1H-SAT ${ }^{a}$

\begin{tabular}{lc}
\multicolumn{1}{c}{ species } & binding energy $E_{\text {bind }}(\mathrm{eV} / \mathrm{H})$ \\
\hline 2H-SAT & -0.46 \\
2H-SAT, hydrogens rotated & $-\mathbf{0 . 0 7}$ \\
1H-SAT & -0.51 \\
1H-SAT, hydrogen rotated & $-\mathbf{0 . 1 3}$ \\
\hline
\end{tabular}

${ }^{a_{T}}$ The energy is always calculated with respect to Ce-TPP $/ \mathrm{Ag}(111)$ and the hydrogen atoms in $\mathrm{H}_{2}$ in the gas phase. The positions of the hydrogen atoms correspond to $2 \mathrm{H}$-TPP tautomer 1 for $2 \mathrm{H}$-SAT and to tautomer $\mathbf{1}^{*}$ for $2 \mathrm{H}$-SAT with rotated hydrogens. Bold values are energetically favored.

well as the two possible Ce-TPP adsorption configurations. As 286 expected from tautomerization experiments, ${ }^{48,51,54}$ the tauto- 287 mers are close in energy $(\Delta E \sim 80 \mathrm{meV})$. In Ce-TPP, the 288 cerium atom cannot be simply incorporated into the macro- 289 cycle plane due to the relatively large size of the metal ion, 290 substantially exceeding atomic diameters of transition metals. ${ }^{32} 291$ This results in two possible interfacial bonding configurations: 292 either with the cerium atom pointing toward the surface (Ce 293 down) or away from it (Ce up) relative to the molecular plane/ 294 macrocycle. From STM and STS data, the orientation cannot 295 be unambiguously identified, although most findings support 296 the Ce down situation (see below). Also the adsorption 297 energies obtained from DFT optimized structures clearly favor 298 Ce down with $5.09 \mathrm{eV} /$ molecule compared to $3.46 \mathrm{eV} / 299$ molecule for $\mathrm{Ce}$ up and therefore substantiate considerations 300 based on experimental data.

Table 2 contains binding energies of the precursor and 302 intermediate SAT complexes. The binding energies represent 303 

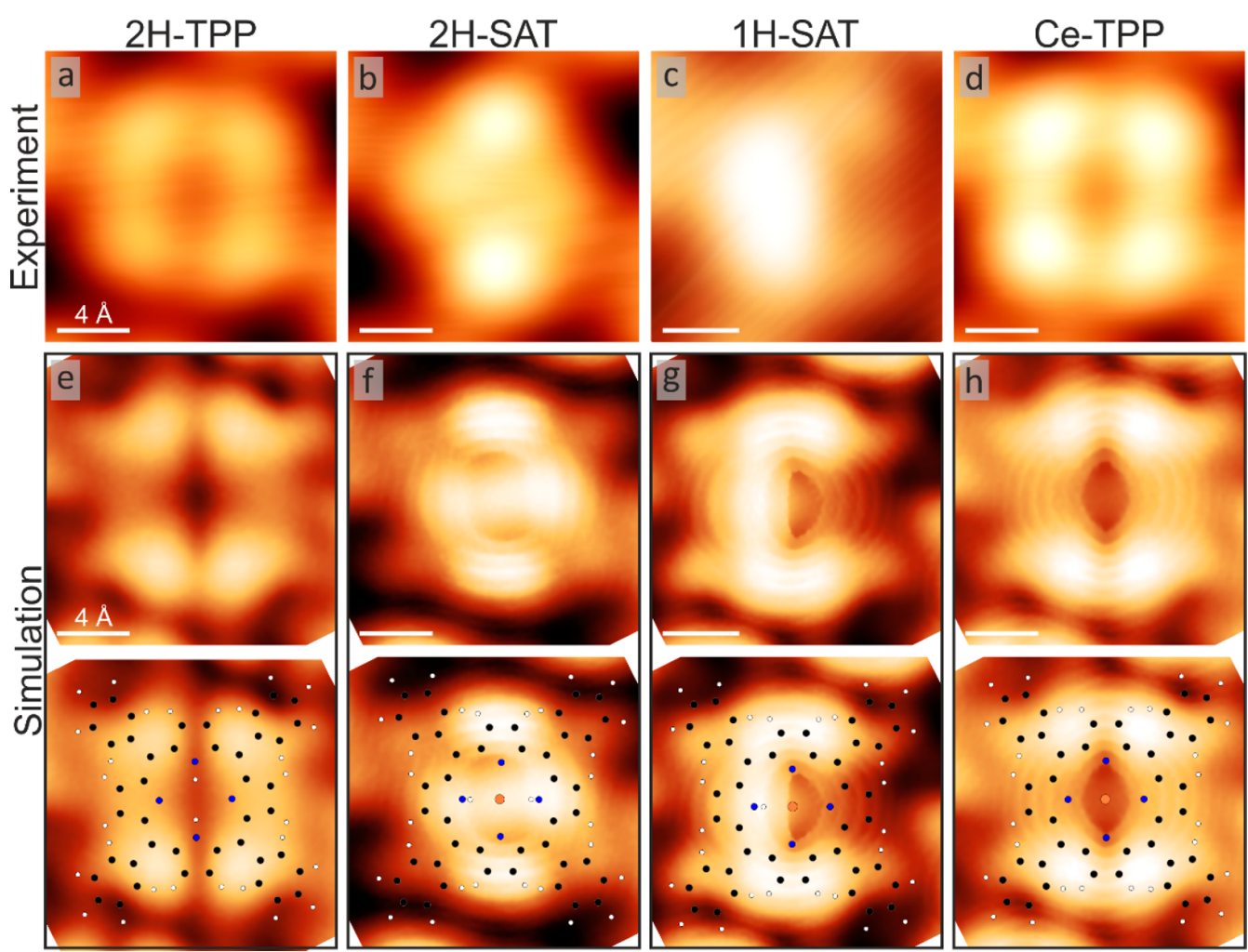

Figure 6. Comparison of experimental with simulated STM data. The first row $(a-d)$ shows the topography of each species as indicated above each column. In parts $\mathrm{e}-\mathrm{h}$, the corresponding STM image simulations are reproduced, whereby the lower panels include the atomic positions. Bias voltages for simulations were chosen according to the STM experiments, i.e., 1.25 (a, b, d) and $1.0 \mathrm{~V}$ (c).

Table 3. Geometric Parameters Extracted from DFT Optimized Adsorption Geometries ${ }^{a}$

$\begin{array}{llllcc} & 2 \text { H-TPP } & \text { 2H-SAT } & \text { 1H-SAT } & \text { Ce-TPP (Ce-down) } & \text { 2H-TPP (gas phase) } \\ \theta_{\text {phenyl }}(\text { deg }) & 29 & 33 & 33 & 32 & 61 \\ \rho_{\text {macro }}(\mathrm{deg}) & -14,21 & -21,28 & -17,26 & -14,24 & -6,6 \\ \Delta z_{\text {Ce-mc }}(\AA) & - & -1.37 & -1.30 & -1.19 & -5,15 \\ \Delta z_{\text {mc }}(\AA) & 3.51 & 3.93 & 3.87 & 3.79 & 0.10 \\ \Delta z_{\text {Ce-s }}(\AA) & - & 2.56 & 2.57 & 2.60 & \end{array}$

${ }^{a} \theta_{\text {phenyl }}$ is the dihedral angle of the phenyl groups, where 0 means a planar alignment, i.e. parallel to the macrocycle plane. $\rho_{\text {macro }}$ is the tilt of the pyrrole groups from the macrocycle plane, where 0 means planar. The two values each give the average over the two pyrroles with alike orientation (bending up- or downward, i.e. the nitrogen pointing towards the surface or away from it). While the pyrrole tilt agrees reasonably well with experimental values extracted from NEXAFS, ${ }^{48}$ the dihedral angle of the phenyl groups is lower than the reported one, which might be attributed to packing effects. $\Delta z_{\mathrm{Ce}-\mathrm{mc}}$ is the height of Ce above $(+)$ or below $(-)$ the macrocycle. $\Delta z_{\mathrm{mc}}$ is the height of macrocycle above the first substrate layer. $\Delta z_{\text {Ce-s }}$ is the height of Ce above the first substrate layer.

304 relative values compared to $\mathrm{Ce}-\mathrm{TPP} / \operatorname{Ag}(111)$ and hydrogen as 305 gas phase $\mathrm{H}_{2}$. All these energies are negative, meaning that the 306 ground state is indeed the desorbed $\mathrm{H}_{2}$ and $\mathrm{Ce}-\mathrm{TPP} / \mathrm{Ag}(111)$, 307 or that $2 \mathrm{H}-\mathrm{Ce}-\mathrm{TPP} / \mathrm{Ag}(111)$ (2H-SAT) and H-Ce-TPP/ $308 \mathrm{Ag}(111)$ (1H-SAT) are metastable states. Furthermore, the 309 binding energies for SAT tautomers were calculated. Surpris310 ingly, for both SAT species, the rotated tautomer configurations $3111^{*}$-not favored for $2 \mathrm{H}$-TPP—are preferred by $\sim 400 \mathrm{meV} / \mathrm{H}$. 312 Consequently, according to our DFT results, the hydrogen 313 positions should change with the evolution of the $\mathrm{Ce}$ 314 tetrapyrrole coordination sphere. A rotation of the whole 315 molecule "on top" of the cerium atom seems unlikely, because 316 of the good agreement of simulated STM images of DFT 317 geometries of tautomer $1^{*}$ configurations with experimental 318 data (see below). Furthermore, the incorporation of the 319 molecules into organic arrays results in interlocking of the 320 peripheral phenyls hindering rotational motion, which is supported by STM manipulation experiments at $\mathrm{Ce}(\mathrm{TPP})_{2} 321$ double and $\mathrm{Ce}_{2}(\mathrm{TPP})_{3}$ triple deckers. ${ }^{12}$ The rotation of TPP 322 moieties is blocked in densely packed $\mathrm{Ce}(\mathrm{TPP})_{2}$ arrays and 323 only possible for the topmost TPP of $\mathrm{Ce}_{2}(\mathrm{TPP})_{3}$ without direct 324 neighbors protruding from lower layers.

For the energetically favored species, simulated STM images 326 were generated. Figure 6 presents a comparison of the $327 \mathrm{f} 6$ simulation to experimental data for each species, whereby key 328 characteristics nicely match. 2H-TPP and Ce-TPP appear alike 329 exhibiting four lobes. Furthermore, the larger apparent height 330 of Ce-TPP with respect to $2 \mathrm{H}$-TPP is reproduced qualitatively 331 (cf. Figure 6a,e to Figure 6d,h). The good agreement allows in 332 particular the determination of molecular species and molecular 333 features for 2H-SAT and 1H-SAT. In STM, 2H-SAT features 334 two prominent lobes defining a main axis. Close to the middle 335 of the main axis two dimmer lobes are located at the left and 336 right (also compare species $\mathbf{2}$ in Figure 3a and Figure 4a) 337 
338 forming a cross-like appearance with a longer side along the 339 main axis. The simulated images express very similar features 340 allowing the assumption that the inner hydrogens of $2 \mathrm{H}$-SAT 341 are situated at different nitrogen atoms compared to $2 \mathrm{H}$-TPP. 342 The prominent lobes in 2H-SAT are observed at the upward 343 bent pyrroles of the TPP macrocycle (i.e., the outer $\mathrm{C}-\mathrm{C}$ bond 344 is highest and the nitrogens point toward the surface) and do 345 not correspond to the hydrogen positions. This stands in 346 contrast to $\mathrm{TPP} / \operatorname{Ag}(111)$ where protrusions are a unique 347 signature of the hydrogen positions in $2 \mathrm{H}-\mathrm{TPP} / \mathrm{Ag}(111)$ and $3481 \mathrm{H}-\mathrm{TPP} / \mathrm{Ag}(111) .{ }^{48}$ Nevertheless, the two dimmer lobes do 349 coincide with the hydrogen binding sites and could be related 350 to an electronic effect. 1H-SAT adopts a C-like shape in STM. 351 This asymmetry is reproduced in the simulations as a uniformly 352 appearing C-shape. Note that there is an additional bright 353 protrusion on the C in STM data at the hydrogen position. It 354 remains unclear whether this feature is an electronic effect 355 caused by $\mathrm{H}$-binding since it is not reproduced theoretically. 356 A geometric effect due to molecular tilting on top of the 357 cerium atom in 1H-SAT cannot be ruled out. Although an 358 evaluation of DFT data for macrocycle tilts only gave angles $359 \leq 2.5^{\circ}$, the absolute height difference of the $\mathrm{N}-\mathrm{H}$ nitrogen and 360 the opposing $\mathrm{N}$ amounts to $\Delta z_{\mathrm{NH}-\mathrm{N}}=0.18 \AA$, thus enough to 361 cause considerable changes in the tunneling current.

362 Table 3 shows geometric parameters for all species that allow 363 following conformational changes induced by the interaction 364 with cerium (angles defined as in Figure 5). Generally, when 365 describing tilt angles of molecular moieties within TPP, a 366 correlation of the pyrrole and phenyl tilts is established that is 367 determined by steric constraints. In gas phase the $2 \mathrm{H}$-TPP 368 macrocycle is almost flat (small tilt $\rho_{\text {macro }}$ of pyrrole groups)

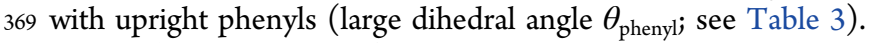
370 Upon adsorbing on a surface, the phenyls are rotated to adopt a 371 more flat orientation ( $\theta_{\text {phenyl }}$ is decreased) due to attractive 372 interactions with the substrate. This rotation results in a 373 deformation of the macrocycle through steric constraints of the 374 phenyls with the pyrroles. ${ }^{55}$ The pyrroles rotate pairwise out of 375 the macrocycle plane; two opposing ones toward the surface 376 (nitrogen points to the surface) and the other two away 377 (nitrogen points toward vacuum). Note that for 2H-TPP/ $378 \mathrm{Ag}(111)$ the aminic pyrroles (including the $\mathrm{N}-\mathrm{H}$ group) rotate 379 down (cf. Figure S1).

380 Metalation of TPP with $3 d$ metals often triggers a flattening 381 of the macrocycle, accompanied by more upright phenyl legs, 382 i.e., a conformation that is more like in the gas phase. 383 Examining the geometric parameters in Table 3, the metalation 384 of 2H-TPP with cerium does not trigger conformational 385 adaptations established for transition metals and moreover 386 changes do not follow the usual phenyl/pyrrole correlation 387 (larger $\rho_{\text {macro, }}$ smaller $\left.\theta_{\text {phenyl }}\right){ }^{56}$ Instead, the macrocycle is 388 deformed more strongly (second row, larger $\rho_{\text {macro }}$ ) accom389 panied by more upright phenyl legs (row 1, larger $\theta_{\text {phenyl }}$ ), not 390 only for the precursor and intermediate, but also for the fully 391 metalated Ce-TPP. This behavior is associated with the 392 lanthanide atomic diameter preventing from a complete 393 macrocycle incorporation. Interestingly, the height $\Delta z_{\text {Ce-s }}$ of 394 the cerium atom above the substrate correlates with the pyrrole 395 tilt angle (cf. rows 2 and 5 in Table 3). The higher the Ce is 396 and the closer it approaches the macrocycle (see $\Delta z_{\text {Ce-m }}$ in row 3973 ), the flatter becomes the latter. Furthermore, $\Delta z_{\text {Ce-s }}$ increases 398 and $\Delta z_{\text {Ce-m }}$ decreases during metalation. This allows the 399 following conclusions for geometric adaptations during the 400 metalation process: The interaction of $2 \mathrm{H}$-TPP with cerium atoms in the precursor complex causes a strong macrocycle 401 distortion that dominates the STM image contrast (see above). 402 Upon coordination, the Ce center is pulled toward the 403 macrocycle and allows for a flatter pyrrole orientation. 404 However, due to its size, it cannot be incorporated fully and 405 hence, the macrocycle can neither flatten out more, as observed 406 for transition metals. Indeed, the final $\mathrm{Ce}-$ macrocycle 407 configuration in Ce-TPP $/ \operatorname{Ag}(111)$ still implies a slightly 408 stronger deformation compared to $2 \mathrm{H}-\mathrm{TPP} / \mathrm{Ag}(111)$ for one 409 of the pyrroles.

\section{DISCUSSION}

As noted in the Introduction, for the vast majority of interfacial 412 tetrapyrrole metalation studies $3 d$ transition metals were 413 employed. Metalation reactions with other classes of metals 414 such as lanthanides are well-known from solution-based 415 chemistry and were recently explored for ultrathin tetrapyrrole 416 films. ${ }^{12,13}$ Unfortunately, the experimental and theoretical 417 results presented above are not fully consistent with a previous 418 interpretation of STM observations. ${ }^{32}$ Therein, a distinct 419 species appearing after Ce exposure of a mixed $2 \mathrm{H}-\mathrm{TPP} / \mathrm{Co}-420$ TPP matrix on $\operatorname{Ag}(111)$ evolved, that is now ascribed to Co- 421 TPP interacting with Ce (cf. Supporting Information, Figure 422 S4), which formation seems to be favorable. Since complexes 423 with the previously reported characteristics were never 424 observed in experiments employing pure $2 \mathrm{H}$-TPP layers, the 425 earlier assignment must be revised (for more details please refer 426 to the Supporting Information). Thus, the present study 427 clarifies the mechanism of on-surface lanthanide tetrapyrrole 428 synthesis in the monolayer regime.

429

The interaction of Ce atoms with $2 \mathrm{H}$-TPP arrays results in 430 changes within the organic layers that resemble well- 431 documented metalation reactions with transition metals., ${ }^{3,4,11,24} 432$ Namely, the island order is not perturbed, but changes occur at 433 the single-molecule level within the arrays, and the number of 434 modified molecules correlates with the amount of deposited 435 metal. A marked difference to transition metals manifests in the 436 coordination geometry of the final metalloporphyrin and stems 437 from the significantly larger size of the lanthanide. DFT 438 calculations showed that the Ce ion cannot be centered in the 439 inner cavity. ${ }^{32}$ Instead it is placed approximately $1 \AA$ out of the 440 macrocycle plane $(\sim 1 \AA$ in gas phase calculation, $0.86 \AA$ above 441 the macrocycle for the Ce up configuration and $1.19 \AA$ A below 442 the macrocycle for Ce down), i.e., the resulting on-surface 443 complex can exhibit two different arrangements: the Ce atom 444 can be located above or below the macrocycle of TPP, but the 445 direct identification by STM is not straightforward. In the top 446 configuration, the Ce might be detectable by STM if 447 appropriate electronic states are available. Indeed, surface- 448 confined, coordinated $\mathrm{Ce}$ atoms and other lanthanides can be 449 imaged as prominent protrusions, ${ }^{57-60}$ however there are also 450 examples where lanthanide coordination centers remain hidden 451 in STM images. ${ }^{57,61-63}$ For comparison, considering the case of 452 Sn-Pc, the metal center can be positioned above or below the 453 molecular plane, and these two molecular conformations can be 454 reversibly switched. ${ }^{64-66}$ By contrast, such characteristics were 455 not observed in our experiments.

Even if the position of the Ce center cannot be determined 457 conclusively in the experiment, the combined STM-DFT 458 analysis clearly signals that the Ce center points toward the 459 $\mathrm{Ag}(111)$ substrate. Furthermore, the formation of TPP-Ce- 460 TPP sandwich structures in the TPP (sub-)monolayer regime 461 was only rarely observed and was not reliably reproducible even 462 
463 at sample temperatures where complexation readily occurs. ${ }^{12}$ In 464 order to synthesize Ce(TPP) $)_{2}$ sandwich complexes, cerium has 465 to be evaporated onto TPP thin films. ${ }^{12}$ This indicates that the 466 Ce center is located below the porphyrin plane, thus preventing 467 the coordination of an additional tetrapyrrole ligand. This 468 interpretation is in agreement with a report from Katoh et al., ${ }^{19}$ 469 where half-sandwich lanthanide-phthalocyanine (Ln-Pc) de470 rivatives were identified as cracking products in the sublimation 471 of a series of double-decker compounds. The corresponding 472 STM/STS analysis suggests that the lanthanide centers in the 473 observed Ln-Pc ( $\mathrm{Ln}=\mathrm{Tb}, \mathrm{Dy}, \mathrm{Y})$ face the employed $\mathrm{Au}(111)$ 474 substrate. In agreement, our DFT calculations suggest a 475 definitive energetic preference for an adsorption configuration 476 with the cerium sandwiched between surface and molecule and 477 the simulated images of the Ce down complex exhibit 478 significant similarity to the experiment.

479 Some metalation reactions do not proceed at room 480 temperature but require elevated temperatures, either to supply 481 metal adatoms (kinetically hindered reaction) or to overcome a 482 formation activation barrier. ${ }^{4}$ Since $2 \mathrm{H}$-SAT complexes are well 483 distributed within organic islands, activation energy apparently 484 is needed for the metalation process itself. A notable spectral 485 signature of successful metalation in TPPs and other 486 tetrapyrroles is a LUMO shifted to higher energies. ${ }^{2,3,11}$ Indeed, 487 as shown in Figure 2a, the LUMO of the proposed Ce-TPP is 488 upshifted by $\approx 100 \mathrm{mV}$ corroborating a metalation reaction 489 occurred. Only very few STS data for SAT complexes exist in 490 literature. For comparison, 2H-TPP sitting above a gold atom 491 on $\mathrm{Au}(111)$ experiences shifts of $500 \mathrm{mV}$ for both HOMO and 492 LUMO. $^{29}$ Following a similar trend for the LUMO, the present 493 2H-SAT species exhibits an upshift of $\approx 400 \mathrm{mV}$. Furthermore, 494 it shows a pronounced peak at $-750 \mathrm{mV}$ that is, however, 495 absent for both $2 \mathrm{H}$-TPP and Ce-TPP. Therefore, this feature is 496 tentatively assigned to a mainly metal-derived contribution of 497 Ce states coupling to the silver surface. Indeed, a hybridization 498 of the central metal ion with the $\mathrm{Ag}(111)$ can lead to electronic 499 states with characteristic energy.

500 The identical features of Ce-induced species following 501 thermal treatment and complexes obtained by STM manipu502 lation allows the following conclusions: (i) The metalation 503 reaction can be triggered both by a voltage pulse from the STM 504 tip or by thermal annealing, (ii) the species with two prominent 505 protrusions (highlighted purple in Figure 1) is indeed the 506 precursor state (2H-SAT) and (iii) the species with only one 507 protrusion is a reaction intermediate, assigned to a $1 \mathrm{H}$-SAT 508 complex. This conclusion is supported by the theoretical 509 evaluation of the energetics of possible species and the 510 calculated STM images. For 2H-TPPs the removal of a 511 hydrogen atom from its macrocycle can manifest as steps in $\mathrm{d} I$ / $512 \mathrm{~d} V$ spectra and as single and double steps in $I(t)$ traces 513 recorded when applying a voltage pulse to a single $2 \mathrm{H}$-TPP 514 molecule. Hence, it is likely that the steps described in Figure $5154 \mathrm{~d}$ are related to deprotonation, respectively to the redox 516 reaction in the case of metalation. Apparently the reaction 517 pathway includes metastable configurations that might be 518 related to the geometry of SAT complexes. DFT calculations 519 suggest a strong macrocycle deformation causing the hydrogens 520 to point away from the macrocyclic plane, i.e., away from the 521 metal center. ${ }^{32}$ The Ce metalation of $2 \mathrm{H}$-TPP layers suggests 522 that the process includes diffusion, intercalation and metalation, 523 and thus exhibits similarities to complexation with transition 524 metals. Moreover, the employment of lanthanides allows clearer 525 identification of precursor and intermediate species. The unambiguous elucidation of the lanthanide-TPP reaction 526 necessitates a reinterpretation of previous results. When 527 depositing Ce onto mixed 2H-/Co-TPP arrays, Ce atoms can 528 intercalate under the array and metalate the free-base species 529 but also bind to Co-TPP, forming $\mathrm{Ce}-\mathrm{Co}-\mathrm{TPP}$ complexes 530 featuring a characteristic electronic signature.

\section{CONCLUSION}

532

In summary, this work presented a detailed experimental and 533 theoretical investigation of the interaction of Ce atoms with 534 surface-confined TPPs. When applying standard protocols 535 known from the metalation of tetrapyrroles with $3 d$ transition 536 metals, Ce engages in a metalation reaction to form Ce-TPP. 537 Ce-TPP was comprehensively characterized with STM, 538 (spatially resolved) STS and computational modeling. Interest- 539 ingly, there is direct evidence for an initial 2H-SAT complex 540 preceding Ce-TPP formation. Furthermore, a 1H-SAT reaction 541 intermediate was identified and characterized directly with 542 submolecular precision. By manipulation experiments the tip- 543 induced on-surface synthesis of intermediate species succeeded, 544 as well as the final Ce-TPP formation from the initial precursor. 545 The real-space analysis, complemented by in-depth theoretical 546 modeling provides insights into the intricate pathways of 547 interfacial lanthanide metalation reactions. 548

Our study thus contributes to the fundamental under- 549 standing of on-surface lanthanide interactions with tetrapyrroles 550 and demonstrates the formation of lanthanide-modified species 551 on surfaces that might provide prospects for heterogeneous 552 catalysis, sensing, quantum engineering, and molecular magnet- 553 ism.

\section{ASSOCIATED CONTENT}

554

S Supporting Information

555

The Supporting Information is available free of charge on the 557 ACS Publications website at DOI: 10.1021/acs.jpcc.7b10363. 558

Details on 2H-TPP adsorption on $\mathrm{Ag}(111)$, visualization 559 of molecular arrangement of $2 \mathrm{H}-\mathrm{TPP} / \mathrm{Ag}(111)$ em- 560 ployed for DFT modeling, visual summary of energetic 561 results from DFT, and STM and STS data covering the 562 interaction of cerium atoms with mixed $\mathrm{Co}-\mathrm{TPP} / 2 \mathrm{H}-563$ TPP arrays on $\operatorname{Ag}(111)$ (PDF)

\section{4}

\section{AUTHOR INFORMATION}

\section{Corresponding Author}

*(W.A) E-mail: wau@tum.de.

ORCID

Felix Bischoff: 0000-0002-4844-6816

Willi Auwärter: 0000-0001-9452-4662

Ari P. Seitsonen: 0000-0003-4331-0650

Johannes V. Barth: 0000-0002-6270-2150

565

\section{Author Contributions}

The manuscript was written through contibutions of 573 authors. All authors have given approval to the final version of 575 the manuscript.

Notes

The authors declare no competing financial interest.

\section{ACKNOWLEDGMENTS}

This work was financially supported by the Munich Center for 580 Advanced Photonics (MAP), TUM-IAS, and the ERC 581 Advanced Grant MolArt (No. 247299). W.A. acknowledges 582 
583 funding by the DFG via a Heisenberg Professorship. We 584 acknowledge fruitful discussions and experimental support from 585 David Écija. A part of the calculations was performed at Centro 586 Svizzero di Calcolo Scientifico (CSCS), Lugano, under Project 587 uzh11.

\section{REFERENCES}

589 (1) Marbach, H. Surface-Mediated in Situ Metalation of Porphyrins 590 at the Solid-Vacuum Interface. Acc. Chem. Res. 2015, 48, 2649-2658. 591 (2) Diller, K.; Papageorgiou, A. C.; Klappenberger, F.; Allegretti, F.; 592 Barth, J. V.; Auwärter, W. In Vacuo Interfacial Tetrapyrrole 593 Metallation. Chem. Soc. Rev. 2016, 45, 1629-1656.

594 (3) Auwärter, W.; Écija, D.; Klappenberger, F.; Barth, J. V. 595 Porphyrins at Interfaces. Nat. Chem. 2015, 7, 105-120.

596 (4) Gottfried, J. M. Surface Chemistry of Porphyrins and 597 Phthalocyanines. Surf. Sci. Rep. 2015, 70, 259-379.

598 (5) Calogero, G.; Bartolotta, A.; Di Marco, G.; Di Carlo, A.; 599 Bonaccorso, F. Vegetable-Based Dye-Sensitized Solar Cells. Chem. Soc. 600 Rev. 2015, 44, 3244-3294.

601 (6) Wang, X.-F.; Tamiaki, H. Cyclic Tetrapyrrole Based Molecules 602 for Dye-Sensitized Solar Cells. Energy Environ. Sci. 2010, 3, 94-106. 603 (7) Qiao, J.; Liu, Y.; Hong, F.; Zhang, J. A Review of Catalysts for the 604 Electroreduction of Carbon Dioxide to Produce Low-Carbon Fuels. 605 Chem. Soc. Rev. 2014, 43, 631-675.

606 (8) Tao, S.; Li, G.; Zhu, H. Metalloporphyrins as Sensing Elements 607 for the Rapid Detection of Trace TNT Vapor. J. Mater. Chem. 2006, $60816,4521-4528$.

609 (9) Écija, D.; Urgel, J. I.; Seitsonen, A. P.; Auwärter, W.; Barth, J. V. 610 Lanthanide-Directed Assembly of Interfacial Coordination Architec611 tures-from Complex Networks to Functional Nanosystems. Acc. 612 Chem. Res. 2018, DOI: 10.1021/acs.accounts.7b00379.

613 (10) Gottfried, J. M.; Flechtner, K.; Kretschmann, A.; Lukasczyk, T.; 614 Steinrück, H.-P. Direct Synthesis of a Metalloporphyrin Complex on a 615 Surface. J. Am. Chem. Soc. 2006, 128, 5644-5645.

616 (11) Auwärter, W.; Weber-Bargioni, A.; Brink, S.; Riemann, A.; 617 Schiffrin, A.; Ruben, M.; Barth, J. V. Controlled Metalation of Self618 Assembled Porphyrin Nanoarrays in Two Dimensions. ChemPhy619 sChem 2007, 8, 250-254.

620 (12) Écija, D.; Auwärter, W.; Vijayaraghavan, S.; Seufert, K.; Bischoff, 621 F.; Tashiro, K.; Barth, J. V. Assembly and Manipulation of Rotatable 622 Cerium Porphyrinato Sandwich Complexes on a Surface. Angew. 623 Chem., Int. Ed. 2011, 50, 3872-3877.

624 (13) Nardi, M.; Verucchi, R.; Tubino, R.; Iannotta, S. Activation and 625 Control of Organolanthanide Synthesis by Supersonic Molecular 626 Beams: Erbium-Porphyrin Test Case. Phys. Rev. B: Condens. Matter 627 Mater. Phys. 2009, 79, 125404.

628 (14) Buchler, J. W.; Decian, A.; Fischer, J.; Kihnbotulinski, M.; 629 Paulus, H.; Weiss, R. Metal Complexes with Tetrapyrrole Ligands. 40. 630 Cerium(Iv) Bis(Octaethylporphyrinate) and Dicerium(III) Tris631 (Octaethylporphyrinate): Parents of a New Family of Lanthanoid 632 Double-Decker and Triple-Decker Molecules. J. Am. Chem. Soc. 1986, $633108,3652-3659$.

634 (15) Buchler, J. W.; Kapellmann, H. G.; Knoff, M.; Lay, K. L.; Pfeifer, 635 S. Metal Complexes with Tetrapyrrole Ligands, XXXI. Neutral and 636 Anionoid Bisporphinates of Cerium and Praeseodymium. Z. 637 Naturforsch., B: J. Chem. Sci. 1983, 38, 1339-1345.

638 (16) Ishikawa, N.; Sugita, M.; Ishikawa, T.; Koshihara, S.; Kaizu, Y. 639 Lanthanide Double-Decker Complexes Functioning as Magnets at the 640 Single-Molecular Level. J. Am. Chem. Soc. 2003, 125, 8694-8695.

641 (17) Ishikawa, N.; Sugita, M.; Ishikawa, T.; Koshihara, S.; Kaizu, Y. 642 Mononuclear Lanthanide Complexes with a Long Magnetization 643 Relaxation Time at High Temperatures: A New Category of Magnets 644 at the Single-Molecular Level. J. Phys. Chem. B 2004, 108, 1126564511271.

646 (18) Bogani, L.; Wernsdorfer, W. Molecular Spintronics Using 647 Single-Molecule Magnets. Nat. Mater. 2008, 7, 179-186.

648 (19) Katoh, K.; Yoshida, Y.; Yamashita, M.; Miyasaka, H.; Breedlove, 649 B. K.; Kajiwara, T.; Takaishi, S.; Ishikawa, N.; Isshiki, H.; Zhang, Y. F.; et al. Direct Observation of Lanthanide(III)-Phthalocyanine Molecules 650 on $\mathrm{Au}(111)$ by Using Scanning Tunneling Microscopy and Scanning 651 Tunneling Spectroscopy and Thin-Film Field-Effect Transistor 652 Properties of $\mathrm{Tb}$ (III)- and Dy(III)-Phthalocyanine Molecules. J. Am. 653 Chem. Soc. 2009, 131, 9967-9976.

(20) Wong, C. P.; Venteicher, Rf; Horrocks, W. D. Lanthanide 655 Porphyrin Complexes - Potential New Class of Nuclear Magnetic- 656 Resonance Dipolar Probe. J. Am. Chem. Soc. 1974, 96, 7149-7150. 657 (21) Duchowski, J. K.; Bocian, D. F. Spectroscopic Characterization 658 of Lanthanide Octaethylporphyrin Sandwich Complexes - Effects of 659 Strong $\pi$ - $\pi$-Interaction. J. Am. Chem. Soc. 1990, 112, 3312-3318. 660

(22) Müllegger, S.; Tebi, S.; Das, A. K.; Schöfberger, W.; Faschinger, 661 F.; Koch, R. Radio Frequency Scanning Tunneling Spectroscopy for 662 Single-Molecule Spin Resonance. Phys. Rev. Lett. 2014, 113, 133001. 663

(23) Cirera, B.; Matarrubia, J.; Kaposi, T.; Gimenez-Agulló, N.; 664 Paszkiewicz, M.; Klappenberger, F.; Otero, R.; Gallego, J. M.; Ballester, 665 P.; Barth, J. V.; et al. Preservation of Electronic Properties of Double- 666 Decker Complexes on Metallic Supports. Phys. Chem. Chem. Phys. 667 2017, 19, 8282-8287.

(24) Shubina, T. E.; Marbach, H.; Flechtner, K.; Kretschmann, A.; 669 Jux, N.; Buchner, F.; Steinrück, H.-P.; Clark, T.; Gottfried, J. M. 670 Principle and Mechanism of Direct Porphyrin Metalation: Joint 671 Experimental and Theoretical Investigation. J. Am. Chem. Soc. 2007, 672 129, 9476-9483.

(25) Shen, Y.; Ryde, U. Reaction Mechanism of Porphyrin 674 Metallation Studied by Theoretical Methods. Chem. - Eur. J. 2005, 675 $11,1549-1564$.

676

(26) Funahashi, S.; Inada, Y.; Inamo, M. Dynamic Study of Metal-Ion 677 Incorporation into Porphyrins Based on the Dynamic Characterization 678 of Metal Ions and on Sitting-Atop Complex Formation. Anal. Sci. 679 2001, 17, 917-927.

680

(27) De Luca, G.; Romeo, A.; Scolaro, L. M.; Ricciardi, G.; Rosa, A. 681 Sitting-Atop Metallo-Porphyrin Complexes: Experimental and The- 682 oretical Investigations on Such Elusive Species. Inorg. Chem. 2009, 48, 683 $8493-8507$.

(28) Li, Y.; Xiao, J.; Shubina, T. E.; Chen, M.; Shi, Z.; Schmid, M.; 685 Steinrück, H.-P.; Gottfried, J. M.; Lin, N. Coordination and Metalation 686 Bifunctionality of $\mathrm{Cu}$ with 5,10,15,20-Tetra(4-Pyridyl)Porphyrin: 687 Toward a Mixed-Valence Two-Dimensional Coordination Network. 688 J. Am. Chem. Soc. 2012, 134, 6401-6408.

689

(29) Mielke, J.; Hanke, F.; Peters, M. V.; Hecht, S.; Persson, M.; 690 Grill, L. Adatoms Underneath Single Porphyrin Molecules on 691 $\mathrm{Au}(111)$. J. Am. Chem. Soc. 2015, 137, 1844-1849. 692

(30) Röckert, M.; Franke, M.; Tariq, Q.; Steinrück, H.-P.; Lytken, O. 693 Evidence for a Precursor Adcomplex During the Metalation of 2HTPP 694 with Iron on $\operatorname{Ag}\left(\begin{array}{lll}1 & 0 & 0\end{array}\right)$. Chem. Phys. Lett. 2015, 635, 60-62. 695

(31) Doyle, C. M.; Krasnikov, S. A.; Sergeeva, N. N.; Preobrajenski, 696 A. B.; Vinogradov, N. A.; Sergeeva, Y. N.; Senge, M. O.; Cafolla, A. A. 697 Evidence for the Formation of an Intermediate Complex in the Direct 698 Metalation of Tetra(4-Bromophenyl)-Porphyrin on the $\mathrm{Cu}(111) 699$ Surface. Chem. Commun. 2011, 47, 12134-12136.

(32) Weber-Bargioni, A.; Reichert, J.; Seitsonen, A. P.; Auwärter, W.; 701 Schiffrin, A.; Barth, J. V. Interaction of Cerium Atoms with Surface- 702 Anchored Porphyrin Molecules. J. Phys. Chem. C 2008, 112, 3453- 703 3455.

704

(33) Vijayaraghavan, S.; Auwärter, W.; Écija, D.; Seufert, K.; Rusponi, 705 S.; Houwaart, T.; Sautet, P.; Bocquet, M.-L.; Thakur, P.; Stepanow, S.; 706 et al. Restoring the Co Magnetic Moments at Interfacial Co-Porphyrin 707 Arrays by Site-Selective Uptake of Iron. ACS Nano 2015, 9, 3605- 708 3616.

(34) Auwärter, W.; Schiffrin, A.; Weber-Bargioni, A.; Pennec, Y.; 710 Riemann, A.; Barth, J. V. Molecular Nanoscience and Engineering on 711 Surfaces. Int. J. Nanotechnol. 2008, 5, 1171.

(35) Createc, D-74391 Erligheim, Germany.

(36) Nečas, D.; Klapetek, P. Gwyddion: An Open-Source Software 714 for Spm Data Analysis. Open Physics 2012, 10, 181-188. 715

(37) Hohenberg, P.; Kohn, W. Inhomogeneous Electron Gas. Phys. 716 Rev. 1964, 136, B864-B871. 
718 (38) Kohn, W.; Sham, L. J. Self-Consistent Equations Including 719 Exchange and Correlation Effects. Phys. Rev. 1965, 140, A1133720 A1138.

721 (39) Giannozzi, P.; Baroni, S.; Bonini, N.; Calandra, M.; Car, R.; 722 Cavazzoni, C.; Ceresoli, D.; Chiarotti, G. L.; Cococcioni, M.; Dabo, I. 723 Quantum Espresso: A Modular and Open-Source Software Project for 724 Quantum Simulations of Materials. J. Phys.: Condens. Matter 2009, 21, 725395502 .

726 (40) Hamada, I. Van Der Waals Density Functional Made Accurate. 727 Phys. Rev. B: Condens. Matter Mater. Phys. 2014, 89, 121103.

728 (41) Dal Corso, A. Pseudopotentials Periodic Table: From H to Pu. 729 Comput. Mater. Sci. 2014, 95, 337-350.

730 (42) Auwärter, W.; Seufert, K.; Klappenberger, F.; Reichert, J.; 731 Weber-Bargioni, A.; Verdini, A.; Cvetko, D.; Dell'Angela, M.; 732 Floreano, L.; Cossaro, A.; et al. Site-Specific Electronic and Geometric 733 Interface Structure of Co-Tetraphenyl-Porphyrin Layers on $\mathrm{Ag}(111)$. 734 Phys. Rev. B: Condens. Matter Mater. Phys. 2010, 81, 245403.

735 (43) Tersoff, J.; Hamann, D. R. Theory of the Scanning Tunneling 736 Microscope. Phys. Rev. B: Condens. Matter Mater. Phys. 1985, 31, 805737813.

738 (44) Seufert, K.; Auwärter, W.; García de Abajo, F. J.; Écija, D.; 739 Vijayaraghavan, S.; Joshi, S.; Barth, J. V. Controlled Interaction of 740 Surface Quantum-Well Electronic States. Nano Lett. 2013, 13, 61307416135 .

742 (45) Buchner, F.; Kellner, I.; Hieringer, W.; Görling, A.; Steinrück, 743 H.-P.; Marbach, H. Ordering Aspects and Intramolecular Conforma744 tion of Tetraphenylporphyrins on $\operatorname{Ag}(111)$. Phys. Chem. Chem. Phys. 745 2010, 12, 13082-13090.

746 (46) Rojas, G.; Chen, X.; Bravo, C.; Kim, J.-H.; Kim, J.-S.; Xiao, J.; 747 Dowben, P. A.; Gao, Y.; Zeng, X. C.; Choe, W.; et al. Self-Assembly 748 and Properties of Nonmetalated Tetraphenyl-Porphyrin on Metal 749 Substrates. J. Phys. Chem. C 2010, 114, 9408-9415.

750 (47) Rojas, G.; Simpson, S.; Chen, X.; Kunkel, D. A.; Nitz, J.; Xiao, J.; 751 Dowben, P. A.; Zurek, E.; Enders, A. Surface State Engineering of 752 Molecule-Molecule Interactions. Phys. Chem. Chem. Phys. 2012, 14, 7534971.

754 (48) Auwärter, W.; Seufert, K.; Bischoff, F.; Écija, D.; Vijayaraghavan, 755 S.; Joshi, S.; Klappenberger, F.; Samudrala, N.; Barth, J. V. A Surface756 Anchored Molecular Four-Level Conductance Switch Based on Single 757 Proton Transfer. Nat. Nanotechnol. 2012, 7, 41-46.

758 (49) Hennig, J.; Limbach, H. H. Kinetic Study of Hydrogen 759 Tunnelling in Meso-Tetraphenylporphine by Nuclear Magnetic 760 Resonance Lineshape Analysis and Selective T1 $\rho$ - Relaxation Time 761 Measurements. J. Chem. Soc., Faraday Trans. 2 1979, 75, 752-766.

762 (50) Limbach, H. H.; Hennig, J. Quasiclassical Calculations of One763 Dimensional Potential Parameters of the Hydrogen Migration in 764 Meso-Tetraphenylporphine from Experimental Tunnel Rates. J. Chem. 765 Phys. 1979, 71, 3120-3124.

766 (51) Liljeroth, P.; Repp, J.; Meyer, G. Current-Induced Hydrogen 767 Tautomerization and Conductance Switching of Naphthalocyanine 768 Molecules. Science 2007, 317, 1203-1206.

769 (52) Garnica, M.; Schwarz, M.; Ducke, J.; He, Y.; Bischoff, F.; Barth, 770 J. V.; Auwärter, W.; Stradi, D. Comparative Study of the Interfaces of 771 Graphene and Hexagonal Boron Nitride with Silver. Phys. Rev. B: 772 Condens. Matter Mater. Phys. 2016, 94, 155431.

773 (53) Comanici, K.; Buchner, F.; Flechtner, K.; Lukasczyk, T.; 774 Gottfried, J. M.; Steinrück, H.-P.; Marbach, H. Understanding the 775 Contrast Mechanism in Scanning Tunneling Microscopy (STM) 776 Images of an Intermixed Tetraphenylporphyrin Layer on $\operatorname{Ag}(111)$. 777 Langmuir 2008, 24, 1897-1901.

778 (54) Kumagai, T.; Hanke, F.; Gawinkowski, S.; Sharp, J.; Kotsis, K.; 779 Waluk, J.; Persson, M.; Grill, L. Controlling Intramolecular Hydrogen 780 Transfer in a Porphycene Molecule with Single Atoms or Molecules 781 Located Nearby. Nat. Chem. 2014, 6, 41-46.

782 (55) Wölfle, T.; Görling, A.; Hieringer, W. Conformational Flexibility 783 of Metalloporphyrins Studied by Density-Functional Calculations. 784 Phys. Chem. Chem. Phys. 2008, 10, 5739-5742.

785 (56) Diller, K.; Klappenberger, F.; Marschall, M.; Hermann, K.; 786 Nefedov, A.; Wöll, Ch.; Barth, J. V. Self-Metalation of $2 \mathrm{H}-$
Tetraphenylporphyrin on $\mathrm{Cu}(111)$ : An X-Ray Spectroscopy Study. J. 787 Chem. Phys. 2012, 136, 014705.

(57) Cirera, B.; Dordević, L.; Otero, R.; Gallego, J. M.; Bonifazi, D.; 789 Miranda, R.; Écija, D. Dysprosium-Carboxylate Nanomeshes with 790 Tunable Cavity Size and Assembly Motif through Ionic Interactions. 791 Chem. Commun. 2016, 52, 11227-11230.

(58) Écija, D.; Urgel, J. I.; Papageorgiou, A. C.; Joshi, S.; Auwärter, 793 W.; Seitsonen, A. P.; Klyatskaya, S.; Ruben, M.; Fischer, S.; 794 Vijayaraghavan, S.; et al. Five-Vertex Archimedean Surface Tessellation 795 by Lanthanide-Directed Molecular Self-Assembly. Proc. Natl. Acad. Sci. 796 U. S. A. 2013, 110, 6678-6681.

(59) Urgel, J. I.; Écija, D.; Auwärter, W.; Barth, J. V. Controlled 798 Manipulation of Gadolinium-Coordinated Supramolecules by Low- 799 Temperature Scanning Tunneling Microscopy. Nano Lett. 2014, 14, 800 1369-1373.

(60) Urgel, J. I.; Écija, D.; Auwärter, W.; Papageorgiou, A. C.; 802 Seitsonen, A. P.; Vijayaraghavan, S.; Joshi, S.; Fischer, S.; Reichert, J.; 803 Barth, J. V. Five-Vertex Lanthanide Coordination on Surfaces: A Route 804 to Sophisticated Nanoarchitectures and Tessellations. J. Phys. Chem. C 805 2014, 118, 12908-12915.

(61) Lyu, G.; Zhang, Q.; Urgel, J. I.; Kuang, G.; Auwärter, W.; Écija, 807 D.; Barth, J. V.; Lin, N. Tunable Lanthanide-Directed Metal- 808 losupramolecular Networks by Exploiting Coordinative Flexibility 809 through Ligand Stoichiometry. Chem. Commun. 2016, 52, 1618-1621. 810

(62) Urgel, J. I.; Cirera, B.; Wang, Y.; Auwärter, W.; Otero, R.; 811 Gallego, J. M.; Alcamí, M.; Klyatskaya, S.; Ruben, M.; Martín, F.; et al. 812 Surface-Supported Robust 2D Lanthanide-Carboxylate Coordination 813 Networks. Small 2015, 11, 6358-6364.

(63) Urgel, J. I.; Écija, D.; Lyu, G.; Zhang, R.; Palma, C. A.; Auwärter, 815 W.; Lin, N.; Barth, J. V. Quasicrystallinity Expressed in Two- 816 Dimensional Coordination Networks. Nat. Chem. 2016, 8, 657-662. 817

(64) Wang, Y. F.; Kröger, J.; Berndt, R.; Hofer, W. A. Pushing and 818 Pulling a Sn Ion through an Adsorbed Phthalocyanine Molecule. J. Am. 819 Chem. Soc. 2009, 131, 3639-3643.

(65) Stadler, C.; Hansen, S.; Kröger, I.; Kumpf, C.; Umbach, E. 821 Tuning Intermolecular Interaction in Long-Range-Ordered Submo- 822 nolayer Organic Films. Nat. Phys. 2009, 5, 153-158.

(66) Baran, J. D.; Larsson, J. A. Structure and Energetics of 824 Shuttlecock-Shaped Tin-Phthalocyanine on $\operatorname{Ag}(111)$ : A Density 825 Functional Study Employing Dispersion Correction. J. Phys. Chem. C 826 2012, 116, 9487-9497.

(67) Flechtner, K.; Kretschmann, A.; Steinrück, H.-P.; Gottfried, J. 828 M. NO-Induced Reversible Switching of the Electronic Interaction 829 between a Porphyrin-Coordinated Cobalt Ion and a Silver Surface. J. 830 Am. Chem. Soc. 2007, 129, 12110-12111.

(68) Hieringer, W.; Flechtner, K.; Kretschmann, A.; Seufert, K.; 832 Auwärter, W.; Barth, J. V.; Görling, A.; Steinrück, H.-P.; Gottfried, J. 833 M. The Surface Trans Effect: Influence of Axial Ligands on the Surface 834 Chemical Bonds of Adsorbed Metalloporphyrins. J. Am. Chem. Soc. 835 2011, 133, 6206-6222.

(69) Liao, M.-S.; Scheiner, S. Electronic Structure and Bonding in 837 Metal Porphyrins, Metal $=\mathrm{Fe}, \mathrm{Co}, \mathrm{Ni}, \mathrm{Cu}, \mathrm{Zn}$. J. Chem. Phys. 2002, 838 $117,205-219$. 